Original

\title{
Analysis of Gene Expression Profile on Uterine Tumorigenesis Initiated with $N$-ethyl- $N$-nitrosourea and Inhibited by Ethinylestradiol in rasH2 Mice
}

\author{
Takao Watanabe ${ }^{1}$, Kayo Sumida ${ }^{2}$, Tomoko Muto ${ }^{1}$, Yoko Kashida ${ }^{1}$, \\ Tomoyuki Watanabe ${ }^{3}$, and Kunitoshi Mitsumori ${ }^{1}$ \\ ${ }^{1}$ Laboratory of Veterinary Pathology, Tokyo University of Agriculture and Technology, 3-5-8 Saiwai-cho, Fuchu-city, \\ Tokyo 183-8509, Japan \\ ${ }^{2}$ Environmental Health Science Laboratory, Sumitomo Chemical Co., Ltd, 3-1-98 Kasugade-naka, Konohana-ku, \\ Osaka 554-8558, Japan \\ ${ }^{3}$ Corporate Planning \& Coordination Office, Sumitomo Chemical Co., Ltd, 2-27-1 Shinkawa, Chuo-ku, Tokyo \\ 104-8260, Japan
}

\begin{abstract}
In order to clarify the possible mechanisms underlying the inhibition of uterine tumorigenesis in rasH2 mice carrying a human prototype c-Ha-ras gene when treated with ethinylestradiol (EE), mice were given a single intraperitoneal injection of $120 \mathrm{mg} / \mathrm{kg} N$-ethyl- $N$-nitrosourea (ENU), followed by $2.5 \mathrm{ppm}$ EE for 6 weeks. Many genes involved in estrogen responses and cell proliferation in the uterus of rasH2 mice are activated by treatment with ENU followed by EE. These include genes associated with receptors such as estrogen receptor (ER) alpha, cell cycles such as cyclin E, cyclin dependent kinase (CDK) 4, CDK inhibitors, ubiquitin and smad3, and growth factors such as epidermal growth factor (EGF) receptor, transforming growth factor (TGF) beta, TGF beta receptor and insulin-like growth factor (IGF). In this study, the ENU+EE treated rasH2 mice demonstrated acceleration of estrogen decomposition and down-regulation of the expression of ER alpha. TGF beta, serine/threonine kinase and smad3, which are downstream of the TGF beta signaling pathway, were also down-regulated, indicating signal values such as CDK4 were down-regulated in the ENU+EE treated rasH2 mice. Ubiquitin which indicates CDK inhibitory metabolism was also down-regulated. Finally, several genes involved in growth factors such as EGF receptor, TGF beta receptor and IGF were also down-regulated. On the other hand, the ENU+EE treated ICR mice showed deceleration of estrogen decomposition and up-regulation of the expression of ER alpha, down-regulation of TGF beta, serine/threonine kinase, smad3, CDK4, ubiquitin, EGF receptor, TGF beta receptor and IGF, indicating that these genes appear to act as stimulants of cell proliferation and carcinogenesis. Therefore, we consider that these genes are key genes which are strongly involved in the inhibition of uterine carcinogenesis in ENU-initiated rasH2 mice treated with EE.
\end{abstract}

(J Toxicol Pathol 2004; 17: 155-164)

Key words: mechanism, rasH2 mice, inhibited uterine tumorigenesis, gene expression profile

\section{Introduction}

Estrogen has potent tumor promoting effects in the uterus, liver, and kidney of experimental animals ${ }^{1-3}$. In the rodent uteri in vitro, estrogen stimulates DNA synthesis and cell proliferation in the luminal and glandular epithelia. Although, to date, several approaches to help decipher the signaling pathways involved in uterine carcinogenesis have

Received: 29 March 2004, Accepted: 24 June 2004

Mailing address: Kunitoshi Mitsumori, Laboratory of Veterinary Pathology, Faculty of Agriculture, Tokyo University of Agriculture and Technology, 3-5-8 Saiwai-cho, Fuchu-city, Tokyo 183-8509, Japan

TEL \& FAX: 81-42-367-5771

E-mail: mitsumor@cc.tuat.ac.jp been introduced for rats and mice, links between hormonal carcinogenesis promoted by estrogens and overexpression of related oncogenes have yet to be definitively demonstrated. In our previous studies in which $N$-ethyl- $N$-nitrosourea (ENU) was intraperitoneally given to transgenic mice carrying a human prototype c-Ha-ras gene ( $\mathrm{rasH} 2$ mice), the incidences of uterine adenocarcinomas, atypical hyperplasias and endometrial hyperplasias were significantly increased, compared to those in non-transgenic mice initiated with $\mathrm{ENU}^{4}$. In contrast, the incidences of these uterine proliferative lesions in ENU-initiated rasH2 mice followed by dietary treatment of $2.5 \mathrm{ppm}$ ethinylestradiol (EE) for 26 weeks were completely inhibited, although significantly high incidences of these proliferating lesions were observed in ENU-initiated ICR mice followed by dietary treatment of $2.5 \mathrm{ppm} \mathrm{EE}$ for 26 
weeks ${ }^{5}$. Proliferating cell nuclear antigen (PCNA) positive indices for uterine adenocarcinomas and atypical hyperplasias in ENU+EE treated ICR mice showed high values, but those in rasH2 mice given ENU alone were comparable to the intact uterine epithelium ${ }^{6}$. Moreover, the immunohistochemical expression of the estrogen receptor (ER) alpha in the uterine luminal and glandular epithelia of the ENU + EE treated ICR mice group was moderate to marked in intensity, compared to that in the ENU alone group (slight in intensity), and there was no consistent difference in ER alpha expression in the uterine luminal and glandular epithelia between the ENU + EE and ENU alone groups of rasH2 mice. The purpose of the present study was to clarify the response to $\mathrm{EE}$ in the uterus of rasH2 mice in which proliferative lesions do not develop as a preliminary study to investigate the mechanisms underlying the inhibition of uterine carcinogenesis. The gene expression patterns from uterine tissues in rasH2 mice given $\mathrm{EE}$ for 6 weeks after ENU initiation were examined using a high density gene chip array and compared with those in ICR mice subjected to the same treatment.

\section{Materials and Methods}

\section{Animals and housing}

A total of 6 female, 6 -week-old rasH2 mice were obtained from the Central Institute for Experimental Animals (CIEA: Kawasaki, Japan), and 6 female ICR mice of the same age were obtained from Japan SLC, Inc. (Shizuoka, Japan). Throughout the acclimatization and experimental periods, animals were housed at a maximum of 3 per polycarbonate cage with absorbent hardwood bedding (White Flakes, Charles River Inc, Tokyo, Japan) in an airconditioned animal room (room temperature, $24 \pm 2{ }^{\circ} \mathrm{C}$; relative humidity of $60 \pm 10 \%$; and 12 hours light/12 hours dark lighting cycle). All animals were transferred to clean cages with fresh bedding twice weekly. The mice were quarantined for 2 weeks in the animal room assigned for the study, and only those without any abnormal findings at the end of this acclimatization period were selected for the experimental period. This study was carried out in accordance with the Guide for Animal Experimentation in the National Institute of Health Sciences of Japan.

\section{Chemicals}

$N$-ethyl-N-nitrosourea (CAS No. 759-73-9) and ethinylestradiol (CAS No. 57-63-6) were purchased from Nacalai Tesque, Inc (Kyoto, Japan).

\section{Experimental design}

After the acclimatization period, all female ICR and rasH2 mice were each divided into 2 groups consisting of 3 animals each (groups 1 and 2: ICR mice; groups 3 and 4: rasH2 mice). Groups 1 and 2 received a single intraperitoneal injection of $120 \mathrm{mg} / \mathrm{kg}$ ENU, followed by 0 and $2.5 \mathrm{ppm}$ EE for 6 weeks, respectively (group 1: ENU alone; and group 2: ENU+2.5 ppm EE). The same administration and doses were used for the rasH2 mice (group 3: ENU alone; and group 4: ENU+2.5 ppm EE). All surviving animals were checked for estrous cycles by vaginal smear at 2 weeks before the final sacrifice at week 6 , when they were exsanguinated from the posterior vena cava under ether anesthesia and subjected to autopsy. After measuring the uterine weights, the uteri were frozen in liquid nitrogen for RNA extraction and subjected to the microarray analysis.

\section{RNA isolation}

Total RNA was isolated from the frozen tissue using TRIzol reagent (Invitrogen Corp., Carlsbad, CA) according to the manufacturer's instructions, and purified using RNeasy Maxi Kit (Quiagen, Valencia, CA). Total RNA was prepared from each uterus of ICR or rasH2 mice administered either ENU alone or in combination with 2.5 ppm EE, and equal amounts of total RNA were pooled from each preparation. The former was used as a control.

\section{High density oligonucleotide microarray analysis}

Murine Genome U74AV.2 Arrays, which contained 6,000 probe sets of known genes and 6,500 probe sets of expressed sequence tags (ESTs) were purchased from Affymetrix (Santa Clara, CA). For microarray probing, reverse transcription, second strand synthesis, and probe generation were all accomplished by the standard Affymetrix protocol (Affymetrix) ${ }^{7}$. Briefly, from $10 \mu \mathrm{g}$ of total RNA, the first strand cDNA was synthesized with SuperScript II reverse transcriptase (Invitrogen, Groningen, the Netherlands) and a T7-(dT)24 primer (Amersham Bioscience, Buckinghamshire, UK), and then the double strand cDNA was synthesized with Escherichia coli RNase H, E. coli DNA polymerase I and E. coli DNA ligase (Invitrogen). From the double strand cDNA, biotin-labeled cRNA was prepared using the BioArray HighYield RNA Transcript Labeling Kit (Enzo Diagnostics, Farmingdale, NY, USA). Twenty micrograms of labeled cRNA was fragmented, and the MGU74AV.2 arrays were hybridized as described in the Gene Chip Expression Analysis Technical Manual (Affymetrix). The arrays were stained with RPhycoerythrin Streptoavidin (Molecular Probes, Eugene, OR, USA), and the fluorescence was intensified by the antibody amplification method. The arrays were scanned with a GeneArray scanner (Agilent Technologies, Palo Alto, CA, USA). The derived signal value was globally normalized and targeted to all probe sets equal to 100 before comparative analysis.

\section{Microarray data analysis}

To examine the gene expression differences between ENU alone treatment and ENU+EE treatment, we performed comparison analysis using the Affymetrix data suite system, MAS 5.0. The genes (probe sets) showing greater than a two-fold change in value with a change of the increase (I) or decrease (D) were designated as changed genes. 


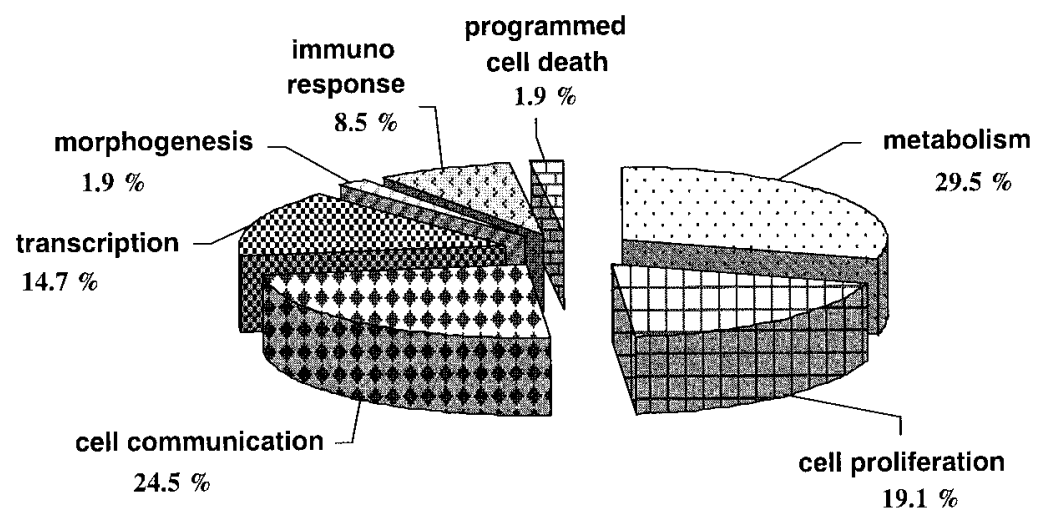

Fig. 1. Pie charts showing gene classification of $>2$-fold up-regulated 1140 genes in the ENU+EE treated rasH2 mice.

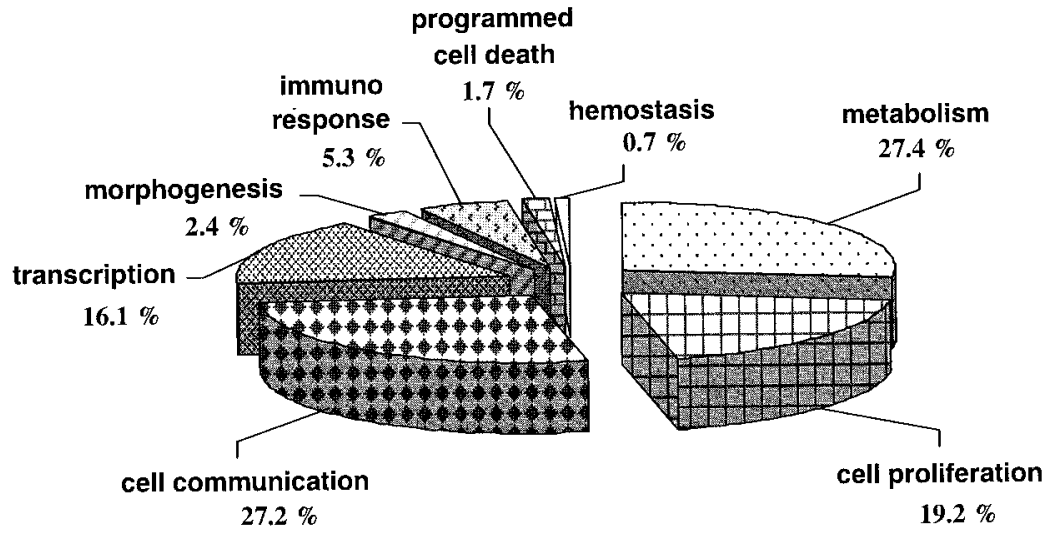

Fig. 2. Pie charts showing gene classification of $<-2$-fold down-regulated 1628 genes in the ENU+EE treated rasH2 mice.

\section{Results}

No animals died during the experimental period. Body weight gains of the ENU+EE group in each strain were depressed throughout the study, although no significant differences in food consumption were observed between the ENU+EE and ENU alone groups. The absolute and relative uterine weights in the ENU+EE group of rasH2 mice were significantly higher than those in the ENU alone group. All mice of both strains in the ENU+EE group showed persistent estrus in the examination of vaginal smears.

Gene expression profiles of uterine tissues in mice treated with ENU+EE are summarized in Figs. 1-4. The genes with unknown function, which were $34-40 \%$ in the expression profiles, were excluded from Figs. 1-4. In the ENU+EE treated rasH2 mice group, the numbers of $>2$-fold up-regulated genes, as compared to the samples from uterine tissues of rasH2 mice of the ENU alone treated group, were 1140 , whereas the numbers of $<-2$-fold down-regulated genes were 1628 (Figs. 1 and 2). When the fold-change criteria was increased to $>3$-fold, 570 and 1965 genes were found to be up-regulated in rasH2 mice and ICR mice, respectively, 775 and 207 genes being down-regulated in rasH2 mice and ICR mice, respectively (data not shown). Specifically, the up-regulated genes in the ENU+EE treated
rasH2 mice group included several genes involved in metabolism such as 11beta-hydroxysteroid dehydrogenase/ carbonyl reductase (average fold change: 2.0), cell growth and maintenance such as $\mathrm{Ca}^{2+}$ dependent activator protein for secretion (3.3-fold), and transcription such as max dimerization protein $(8.1$-fold) (Table 1$)$. The downregulated genes in the ENU+EE treated rasH2 mice group also included several genes involved in metabolism such as hydroxysteroid sulfotransferase (mSTa2) (-2.8-fold), cell growth and maintenance such as inhibition alpha subunit (3.5-fold) (Table 2). In contrast, in the ENU+EE treated ICR mice group, 6567 genes were $>2$-fold up-regulated, and 409 genes were $<-2$-fold down-regulated as compared to the ENU alone treated ICR mice (Figs. 3 and 4). In detail, the up-regulated genes in the ENU+EE treated ICR mice group included several genes involved in metabolism such as hydroxysteroid dehydrogenase 3 (2.9-fold), cell growth and maintenance such as ros proto-oncogene (13.6-fold), and transcription such as homeo box D1 (10.8-fold) (Table 3). The down-regulated genes in the ENU+EE treated ICR mice group also included several genes involved in metabolism such as hydroxysteroid 17-beta dehydrogenase II (-3.6fold), cell growth and maintenance such as mitogenactivated protein kinase kinase (MKK7) (-4.0-fold), and transcription such as IFN-response binding factor 1 (-6.4- 
fold) (Table 4).

As the second step selecting the key genes that are related to the enhanced uterine carcinogenesis in the ENU+EE treated ICR mice and depressed uterine carcinogenesis in the ENU+EE treated rasH2 mice, we selected the candidate genes up-regulated in rasH2 mice and down-regulated in ICR mice or the candidate genes downregulated in rasH2 mice and up-regulated in ICR mice, based

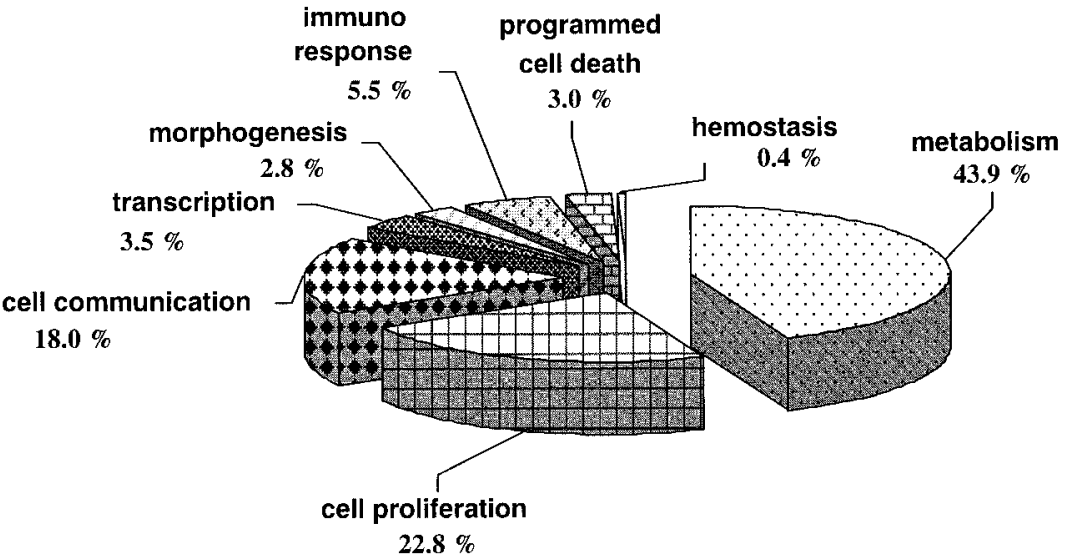

Fig. 3. Pie charts showing gene classification of $>2$-fold up-regulated 6567 genes in the ENU+EE treated ICR mice.

Table 1. Expression Profiles Categorization Based on the Biological Process (up-regulated gene in ENU+EE rasH2 mice)

\begin{tabular}{|c|c|c|c|}
\hline Biological process & Gene name & $\begin{array}{l}\text { Average fold } \\
\text { change }\end{array}$ & $\begin{array}{c}\text { Accession } \\
\text { number }\end{array}$ \\
\hline \multicolumn{4}{|l|}{ Metabolism } \\
\hline \multirow[t]{2}{*}{ Steroid metabolism } & 11 beta-hyhdroxysteroid dehydrogenase/carbonyl reductase & 2.0 & X83202 \\
\hline & 17-beta-hydroxysteroid dehydrogenase type 1 & 3.3 & AF039299 \\
\hline Alcohol metabolism & Aldehyde dehydrogenase II & 4.7 & M74570 \\
\hline Lipid metabolism & Hepatic lipase & 3.8 & X58426 \\
\hline Fatty acid metabolism & Long chain fatty acyl CoA synthetase & 2.4 & U15977 \\
\hline \multirow[t]{2}{*}{ Electron transport } & Cytochrome P450, 2d11 & 3.5 & AI595772 \\
\hline & Cytochrome P450 monooxygenase (CYP2J6) & 2.9 & U62295 \\
\hline \multicolumn{4}{|l|}{ Cell growth and maintenance } \\
\hline Cell growth and maintenance & $\mathrm{Ca}^{2+}$ dependent activator protein for secresion & 3.3 & D86214 \\
\hline Regulation of cell cycle & EGF-like growth factor receptor ErbB4 intracellular domain & 2.0 & AF059177 \\
\hline Ion transport & Potassium channel, subfamily K, member 1 & 3.6 & AF033017 \\
\hline Cation transport & Plasma membrane $\mathrm{Ca}^{2+}$-ATPase 2 & 3.5 & AF053471 \\
\hline Cell proliferation & Protein tyrosine phosphatase, non-receptor type 18 & 2.0 & U49853 \\
\hline \multicolumn{4}{|l|}{ Cell adhesion } \\
\hline \multirow[t]{4}{*}{ Cell adhesion } & CD6 antigen & 12.3 & U37543 \\
\hline & Vascular cell adhesion molecule- 1 truncated form T-VCAM-1 & 2.9 & U12884 \\
\hline & Type 2 desmoglein & 2.7 & AJ000328 \\
\hline & Procollagen, type XVIII, alpha 1 & 2.3 & L22545 \\
\hline \multicolumn{4}{|l|}{ Signal transduction } \\
\hline G-protein coupled receptor signaling & Cannabinoid receptor 2 (macrophage) & 6.0 & X86405 \\
\hline Intercellular signaling cascade & Naturiuretic peptide receptor A & 3.8 & L31932 \\
\hline \multicolumn{4}{|l|}{ Immunity and defence } \\
\hline Immune response & Small inducible cytokine A2 & 3.4 & M19681 \\
\hline Complement activation & Complement factor H-related protein & 2.3 & M29007 \\
\hline \multicolumn{4}{|l|}{ Transcription } \\
\hline \multirow[t]{2}{*}{ Regulation of transcription } & Max dimerization protein & 8.1 & X83106 \\
\hline & Cyclin dependent kinase inhibitor $2 \mathrm{~B}$ & 4.3 & AF059567 \\
\hline \multicolumn{4}{|l|}{ Morphogenesis } \\
\hline Skeletal development & Homeo box D12 & 2.5 & X58849 \\
\hline Neurogenesis & mammalian achaete scute homolog 2 & 3.2 & U77628 \\
\hline \multicolumn{4}{|l|}{ Cell death } \\
\hline Apoptosis & Amyloid beta (A4) precursor-like protein 1 & 2.3 & L04538 \\
\hline
\end{tabular}




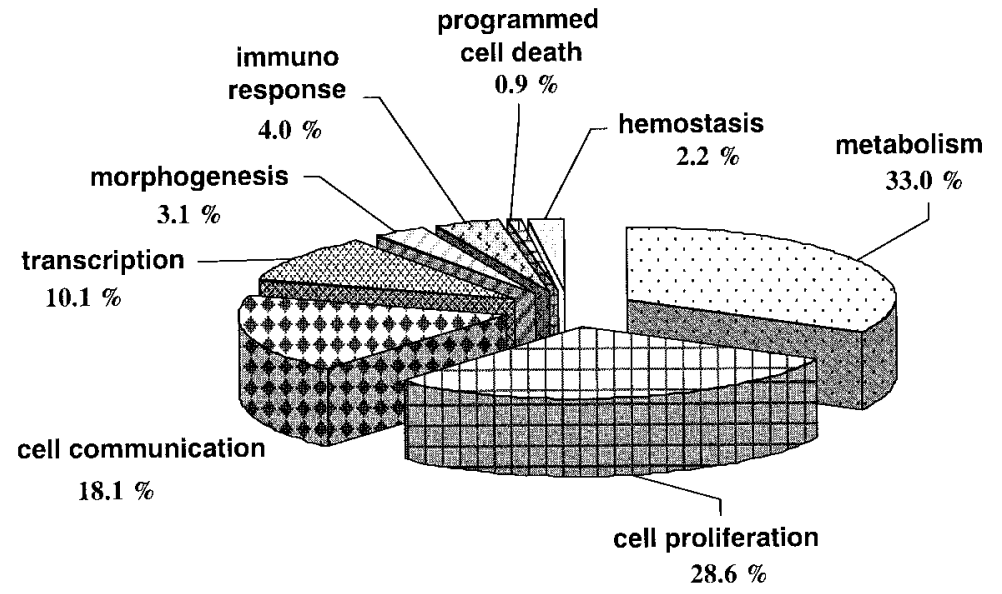

Fig. 4. Pie charts showing gene classification of $<-2$-fold down-regulated 409 genes in the ENU+EE treated ICR mice.

Table 2. Expression Profiles Categorization Based on the Biological Process (down-regulated gene in ENU+EE rasH2 mice)

\begin{tabular}{|c|c|c|c|}
\hline Biological process & Gene name & fold change & Accession number \\
\hline \multicolumn{4}{|l|}{ Metabolism } \\
\hline Steroid metabolism & Hydroxysteroid sulfotransferase (mSTa2) & -2.8 & L27121 \\
\hline Lipid metabolism & Monoglyceride lipase & -2.6 & AJ001118 \\
\hline Amino acid metabolism & Glutamic acid decarboxylase 1 & -3.1 & Z49976 \\
\hline \multirow[t]{2}{*}{ Electron transport } & Cytochrome $\mathrm{P} 450,26$ retinoic acid & -9.8 & Y12657 \\
\hline & CYP 1-b-1 & -7.6 & X78445 \\
\hline \multicolumn{4}{|l|}{ Cell growth and maintenance } \\
\hline Cell growth and maintenance & Inhibin alpha subunit & -3.5 & X55957 \\
\hline \multirow[t]{4}{*}{ Regulation of cell cycle } & Insulin like growth factor binding protein 4 & -3.1 & X76066 \\
\hline & Cyclin B1 & -2.9 & X64713 \\
\hline & Cyclin B2 & -2.3 & X66032 \\
\hline & Cyclin E & -2.0 & X75888 \\
\hline Regulation of cell growth & Insulin-like growth factor binding protein 5 & -3.4 & L12447 \\
\hline \multirow[t]{2}{*}{ Ion transport } & Calcium channel, $\mathrm{P} / \mathrm{Q}$ type, alpha $1 \mathrm{~A}$ & -12.1 & U76716 \\
\hline & Glutamate receptor, ionotropic, NMDA2B (epsilon 2) & -4.7 & D10651 \\
\hline Cation transport & Potassium channel Kv4.2 & -22.8 & AF 107780 \\
\hline \multirow[t]{2}{*}{ Cell proliferation } & Fibroblast growth factor 6 & -9.9 & M92416 \\
\hline & Hepatocyte growth factor & -5.3 & $\mathrm{X} 72307$ \\
\hline \multirow[t]{2}{*}{ Cell cycle } & Growth arrest specific 2 & -11.0 & M21828 \\
\hline & Cell division cycle 7-like 1 & -2.8 & AB019388 \\
\hline \multirow[t]{2}{*}{ Mitosis } & Cell division cycle control protein $2 \mathrm{a}$ & -4.0 & M38724 \\
\hline & Cyclin A2 & -2.5 & X75483 \\
\hline \multicolumn{4}{|l|}{ Cell adhesion } \\
\hline Cell communication & Connexin-36 & -2.0 & Y14331 \\
\hline \multirow[t]{6}{*}{ Cell adhesion } & Procollagen, type XI alpha 1 & -19.8 & D38162 \\
\hline & Procollagen, type XIII, alpha 1 & -15.8 & U30292 \\
\hline & Procollagen, typeXVIII, alpha 1 & -6.4 & U03715 \\
\hline & Procollagen, typeXV & -4.0 & D17546 \\
\hline & Cadherin 16 & -6.5 & AF016271 \\
\hline & Laminin, alpha 2 & -4.1 & U12147 \\
\hline \multicolumn{4}{|l|}{ Signal transduction } \\
\hline \multirow[t]{2}{*}{ G-protein coupled receptor signaling } & G-protein coupled receptor (EBI1) & -5.5 & L31580 \\
\hline & G-protein signaling 9 (RGS9) & -3.2 & AF011358 \\
\hline \multirow[t]{2}{*}{ Intercellular signaling cascade } & Megakaryocyte-associated tyrosine kinase & -5.6 & D45243 \\
\hline & Growth factor receptor bound protein 10 & -4.4 & U18996 \\
\hline \multicolumn{4}{|l|}{ Immunity and defence } \\
\hline Immune response & Interleukin 10 & -5.1 & M37897 \\
\hline \multicolumn{4}{|l|}{ Morphogenesis } \\
\hline \multirow[t]{3}{*}{ Skeletal development } & Mesenchyme homeobox 2 & -15.0 & Z16406 \\
\hline & Homeo box D1 & -14.0 & M87802 \\
\hline & Homeo box A10 & -4.5 & U08757 \\
\hline Muscle development & Myosin heavy chain, skeletal muscle & -2.4 & M12289 \\
\hline \multicolumn{4}{|l|}{ Cell death } \\
\hline Apoptosis & Bcl-x gene, promoter region & -2.8 & AF088904 \\
\hline
\end{tabular}


Table 3. Expression Profiles Categorization Based on the Biological Process (up-regulated gene in ENU+EE ICR mice)

\begin{tabular}{|c|c|c|c|}
\hline Biological process & Gene name & $\begin{array}{l}\text { Average fold } \\
\text { change }\end{array}$ & $\begin{array}{c}\text { Accession } \\
\text { number }\end{array}$ \\
\hline \multicolumn{4}{|l|}{ Metabolism } \\
\hline \multirow[t]{4}{*}{ Steroid metabolism } & Hydroxysteroid dehydrogenase- 3 & 2.9 & M77015 \\
\hline & Hydroxysteroid dehydrogenase- 4 & 4.0 & L16919 \\
\hline & 17-beta-hydroxysteroid dehydrogenase type 7 & 6.6 & Y15733 \\
\hline & 17-beta-hydroxysteroid dehydrogenase type 3 & 5.0 & U66827 \\
\hline Lipid metabolism & Sterol-C5-desaturase & 2.7 & AB016248 \\
\hline \multirow{2}{*}{ Amino acid metabolism } & Glutamate decarboxylase & 7.5 & D42051 \\
\hline & glycoprotein-associated amino acid transporter & 3.4 & AJ012754 \\
\hline Electron transport & Cytochrome P450, 3a16 & 5.0 & D26137 \\
\hline \multirow[t]{2}{*}{ Carbohydrate metabolism } & Glycoprotein 39 & 9.3 & X93035 \\
\hline & $\mathrm{N}$-acetyl galactosaminidase, alpha & 2.6 & AJ223966 \\
\hline \multicolumn{4}{|l|}{ Cell growth and maintenance } \\
\hline \multirow[t]{4}{*}{ Regulation of cell cycle } & Ros proto-oncogene & 13.6 & X81650 \\
\hline & Interleukin 1-alpha & 4.2 & M14639 \\
\hline & Interleukin 1-beta & 9.3 & M15131 \\
\hline & Transforming growth factor, beta2 & 3.8 & X57413 \\
\hline \multirow[t]{6}{*}{ Regulation of cell growth } & Insulin-like growth factor binding protein 2 & 16.0 & X81580 \\
\hline & Insulin-like growth factor binding protein 10 & 3.5 & M32490 \\
\hline & Fibroblast growth factor & 5.2 & AF065903 \\
\hline & Fibroblast growth factor 5 & 7.5 & M37823 \\
\hline & Fibroblast growth factor 6 & 3.9 & M92416 \\
\hline & Fibroblast growth factor 9 & 2.3 & U33535 \\
\hline \multirow[t]{3}{*}{ Ion transport } & Potassium voltage-gated channel & 8.0 & X60475 \\
\hline & $\mathrm{K}^{+}$channel beta- 1 & 5.6 & AF033003 \\
\hline & Chloride channel protein 3 & 3.9 & AF029347 \\
\hline Cation transport & Transient receptor protein 1 & 17.3 & U73625 \\
\hline Lipid transport & Fatty acid transport protein 4 & 2.9 & AF072759 \\
\hline \multirow{5}{*}{ Cell cycle } & Cyclin-dependent kinase inhibitor p27kip 1 & 5.7 & U09968 \\
\hline & Cyclin-dependent kinase inhibitor $1 \mathrm{~A}(\mathrm{p} 21)$ & 5.0 & U09507 \\
\hline & Cyclin-dependent kinase 5 & 2.7 & D29678 \\
\hline & Cyclin D2 & 3.0 & M83749 \\
\hline & Cyclin G2 & 2.1 & U95826 \\
\hline \multicolumn{4}{|l|}{ Cell adhesion } \\
\hline \multirow[t]{2}{*}{ Cell communication } & Gap junction membrane channel protein beta 2 & 5.0 & M81445 \\
\hline & Gap junction membrane channel protein beta 6 & 15.6 & Z70023 \\
\hline \multirow[t]{3}{*}{ Cell adhesion } & Integrin alpha 6 & 5.9 & X69902 \\
\hline & Laminin, beta 3 & 5.2 & U43298 \\
\hline & Collagen IV alpha 4 chain & 4.6 & Z35167 \\
\hline \multirow[t]{2}{*}{ Cell-cell adhesion } & Vascular cell adhesion molecule 1 & 5.1 & M84487 \\
\hline & Intercellular adhesion molecule 2 & 4.4 & X65493 \\
\hline \multicolumn{4}{|l|}{ Signal transduction } \\
\hline \multirow[t]{2}{*}{ G-protein coupled receptor signaling } & Regulator of G-protein signaling 9 & 7.0 & AF011358 \\
\hline & G-protein coupled receptor & 4.0 & D21062 \\
\hline Intercellular signaling cascade & Protein kinase $\mathrm{C}$ epsilon & 5.1 & AF028009 \\
\hline \multicolumn{4}{|l|}{ Immunity and defence } \\
\hline \multirow[t]{2}{*}{ Immune response } & Fc receptor, $\mathrm{IgG}$, alpha chain transporter & 13.3 & L17022 \\
\hline & Interleukin 10 & 3.6 & M37897 \\
\hline \multirow[t]{2}{*}{ Complement activation } & Complement component 4 binding protein & 26.9 & M17122 \\
\hline & Complement factor H-related protein & 5.4 & M29009 \\
\hline Transcription & & & \\
\hline Regulation of transcription & Homeo box D1 & 10.8 & M87802 \\
\hline Morphogenesis & & & \\
\hline Cellular morphogenesis & Type 1 epidermal keratin & 4.4 & M13805 \\
\hline Cell death & & & \\
\hline Apoptosis & Tnf-receptor-associated factor 1 & 18.5 & L35302 \\
\hline & Apoptosis inhibitor 1 & 4.8 & U88908 \\
\hline & Bcl-2-binding protein & 2.9 & AF022223 \\
\hline & Caspase 7 & 2.4 & U67321 \\
\hline
\end{tabular}


Table 4. Expression Profiles Categorization Based on the Biological Process (down-regulated gene in ENU+EE ICR mice)

\begin{tabular}{|c|c|c|c|}
\hline Biological process & Gene name & $\begin{array}{c}\text { Average fold } \\
\text { change }\end{array}$ & $\begin{array}{l}\text { Accession } \\
\text { number }\end{array}$ \\
\hline \multicolumn{4}{|l|}{ Metabolism } \\
\hline Steroid metabolism & Hydroxysteroid 17-beta dehydrogenase II & -3.6 & Y09517 \\
\hline \multirow[t]{2}{*}{ Electron transport } & Cytochrome P450, steroid inducible $3 \mathrm{a} 11$ & -27.4 & X60452 \\
\hline & Cytochrome P450IIIA25 & -4.1 & Y11995 \\
\hline \multicolumn{4}{|l|}{ Cell growth and maintenance } \\
\hline Regulation of cell cycle & Mitogen-activated protein kinase kinase (MKK7) & -4.0 & AF026216 \\
\hline Ion transport & Gamma-aminobutyric acid (GABA-A) receptor, subunit alpha 6 & -3.2 & X51986 \\
\hline \multirow[t]{2}{*}{ Lipid transport } & Apolipoprotein A-I gene & -11.7 & U79573 \\
\hline & Very-long-chain acyl-CoA synthetase related protein & -4.1 & AJ223959 \\
\hline Cell cycle & P38 delta MAP kinase & -4.9 & U81823 \\
\hline Mitosis & Cell division cycle $25 \mathrm{~A}$ & -3.8 & U27323 \\
\hline \multicolumn{4}{|l|}{ Cell adhesion } \\
\hline \multirow[t]{3}{*}{ Cell adhesion } & Cadherin 16 & -7.7 & AF016271 \\
\hline & Procollagen, type XIII, alpha 1 & -3.4 & U30292 \\
\hline & Laminin, gamma 1 & -3.3 & J03484 \\
\hline \multicolumn{4}{|l|}{ Signal transduction } \\
\hline G-protein coupled receptor signaling & Orphan G protein-coupled receptor FEX & -3.7 & AF110818 \\
\hline Intercellular signaling cascade & Nitric oxide synthase 1 , neuronal & -2.8 & D14552 \\
\hline \multicolumn{4}{|l|}{ Immunity and defence } \\
\hline Immune response & Macrophage interferon inducible protein 10 & -2.4 & M33266 \\
\hline Complement activation & Complement receptor 2 & -5.8 & M29281 \\
\hline \multicolumn{4}{|l|}{ Transcription } \\
\hline \multirow[t]{2}{*}{ Regulation of transcription } & IFN-response binding factor 1 & -6.4 & M55290 \\
\hline & Mesenchyme homeobox 2 & -4.8 & Z16406 \\
\hline
\end{tabular}

Table 5. Representative Common Genes that Fluctuated in Opposite Directions between ENU+EE rasH2 and ENU+EE ICR mice

\begin{tabular}{|c|c|c|c|}
\hline \multirow{2}{*}{ Gene name } & \multicolumn{2}{|c|}{ Average fold change } & \multirow{2}{*}{ Accession number } \\
\hline & rasH2 & ICR & \\
\hline \multicolumn{4}{|l|}{ Estrogen decomposition } \\
\hline Hydroxysteroid 17-beta dehydrogenase II & 7.1 & -3.6 & Y09517 \\
\hline \multicolumn{4}{|l|}{ Estrogen receptor } \\
\hline Estrogen receptor alpha & -1.4 & 2.8 & M38651 \\
\hline \multicolumn{4}{|l|}{ TGF beta/smad signaling pathway } \\
\hline Transforming growth factor beta 1 induced transcript 4 & -1.2 & 2.2 & X62940 \\
\hline Transforming growth factor beta 1 induced factor 2 & -1.3 & 2.8 & AF075717 \\
\hline Transforming growth factor beta type 1 receptor & -1.8 & 2.0 & L15436 \\
\hline Serine/threonine kinase (nek2) & -1.9 & 1.2 & AF013166 \\
\hline Smad 3 & -1.4 & 3.3 & AB008192 \\
\hline \multicolumn{4}{|l|}{ Cell cycle } \\
\hline Cyclin E & -2.0 & 1.2 & X75888 \\
\hline Cyclin dependent kinase 4 & -1.7 & 1.7 & L01640 \\
\hline Cdk4 and Cdk6 inhibitor p18 protein & -1.6 & 1.7 & U19596 \\
\hline Cdk4 and Cdk6 inhibitor p19 protein & -1.3 & 2.2 & U19597 \\
\hline Cyclin-dependent kinase inhibitor 1C (p57) & -1.7 & 1.7 & U22399 \\
\hline Ubiquitin-conjugating enzyme & -1.1 & 2.0 & AJ130961 \\
\hline mutant p53 & -1.3 & 2.1 & AB021961 \\
\hline \multicolumn{4}{|l|}{ Growth factor } \\
\hline Epidermal growth factor receptor (EGFR) & -3.6 & 1.9 & L06864 \\
\hline Transforming growth factor-beta type 1 receptor & -1.8 & 2.0 & L15436 \\
\hline Insulin-like growth factor 1 & -4.3 & 1.5 & X04480 \\
\hline Insulin-like growth factor 2 & -2.6 & 1.9 & X71922 \\
\hline Insulin-like growth factor binding protein 3 & -3.0 & 1.9 & X81581 \\
\hline Insulin like growth factor binding protein 4 & -3.1 & 2.3 & X76066 \\
\hline
\end{tabular}


on the data in Tables 1 to 4 . There were no candidate genes other than hydroxysteroid 17-beta dehydrogenase II among the genes that fluctuated more than 2 -fold. Therefore, we extended the analysis to the genes in which fold changes were smaller than 2. In the ENU+EE treated rasH2 mice, one gene involved in estrogen metabolism, hydroxysteroid 17-beta dehydrogenase II (7.1-fold), was up-regulated, whereas estrogen receptor such as ER alpha (-1.4-fold) was down-regulated (Table 5). Many genes related to transforming growth factor (TGF) beta/smad signaling pathway such as TGF beta 1 induced transcript 4 (-1.2-fold), TGF beta 1 induced factor 2 (-1.3-fold), TGF beta type 1 receptor $(-1.8$-fold), serine/threonine kinase (nek2) (-1.9fold) and smad 3 (-1.4-fold) were down-regulated in rasH2 mice, as shown in Table 5. Other down-regulated genes observed were involved in cell cycles such as cyclin E (-2.0fold), cyclin dependent kinase (CDK) 4 (-1.7-fold), CDK4 and CDK6 inhibitor-p18 protein, -p19 protein $(-1.6,-1.3-$ fold), CDK inhibitor $1 \mathrm{C}$ (p57) (-1.7-fold), ubiquitinconjugating enzyme (-1.1-fold) and mutant p53 (-1.3-fold). Several genes involved in growth factor such as epidermal growth factor receptor (EGFR) (-3.6-fold), transforming growth factor-beta type 1 receptor $(-1.8$-fold), insulin-like growth factor (IGF) -1, -2 (-4.3, -2.6-fold), IGF binding protein-3, $-4(-3.0,-3.1$-fold) were down-regulated genes in rasH2 mice. In contrast, in the ENU+EE treated ICR mice, average fold changes of these genes showed fluctuations opposite to those in rasH2 mice (Table 5).

\section{Discussion}

In the present study, we analyzed mRNA expression of some molecules involved in estrogen metabolism, TGF beta/ smad signaling pathway, cell cycle and growth factor. With regards to estrogen metabolism, hydroxysteroid 17-beta dehydrogenase II (which is known to be involved in the decomposition of estradiol (E2) to estrone) was up-regulated in the ENU+EE treated rasH2 mice compared with the ENU alone treated rasH2 mice, indicating that the decomposition rate of EE is relatively rapid in rasH2 mice. The findings that ER alpha and several transcription factors were downregulated in the ENU+EE treated rasH2 mice, suggest that it is difficult to progress in the direction of transcription and growth stimulation. In the TGF beta/smad signaling pathway, TGF beta (which acts as a suppressor of tumor formation in early stages of carcinogenesis and stimulates invasiveness and metastasis of carcinoma cells in late stages $^{8,9}$ ) was down-regulated in the ENU+EE treated rasH2 mice. An alteration in the effects of TGF beta is caused by changes in TGF beta receptor levels at the cell surface or through epigenetic mechanisms ${ }^{8,9}$. Therefore, it is considered that TGF beta is not involved in the inhibition of uterine tumorigenesis in the ENU+EE treated rasH2 mice especially at an early stage. Serine/threonine kinase and smad3, which are downstream of TGF beta signaling pathway, were also down-regulated in the ENU+EE treated $\mathrm{rasH} 2$ mice, indicating that it is difficult to progress in the direction of transcription. In contrast, in the ENU+EE treated ICR mice, the genes described above were upregulated, indicating that advance in the direction for transcription was accelerated and that these genes were involved in the enhanced uterine tumorigenesis.

It is known that estrogens (E2) and estrone (E1) are metabolized via two major pathways: formation of the 2- and 4-catechol estrogens and 16 alpha-hydroxylation ${ }^{10,11}$. CYP1B1 which is the major enzyme catalyzing the synthesis of 4-catechol estrogen ${ }^{11,12}$ was down-regulated in both strains of the ENU+EE treated group (data not shown). However, the signal value was larger in the ENU+EE treated rasH2 mice (approximately $1 / 7$-fold) than that in the ENU+EE treated ICR mice (approximately1/2-fold), indicating the difficulty of generating malignant tumors in the ENU+EE treated rasH2 mice. With regard to the genes involved in cell cycle processes (cyclin E, cyclin dependent kinase such as CDK4, and CDK inhibitor such as p16INK4A, p18INK4C, p19ARF, and p57KIP2), these were down-regulated in the ENU+EE treated rasH2 mice, whereas these genes were up-regulated in the ENU+EE treated ICR mice. It is known that four important pathways, the p21WAF1-p27KIP1-cyclin E-CDK2, $\beta$-catenin-Tcf-myctelomerase, ARF-MDM2-p53, and pRb1-cyclin D1-CDK4/ 6-p16INK4A, are mediated through the appropriate functioning of cell-cycle mechanisms ${ }^{13,14}$. In the pRb1pathway, each of these components plays either a positive or a negative role in cell-cycle control mechanisms ${ }^{15,16}$. This pathway in uterine endometrial carcinogenesis participates in the regulation of the cellular processes at the transition of G1/S phases of the cell-cycle in humans ${ }^{17}$. The phosphorylation of $\mathrm{pRb} 1$ is generally achieved by cyclin D1 complex with cyclin-dependent kinases (4 and 6) at the G1/ $\mathrm{S}$ checkpoint. Conversely, expression of cyclin D1 is also dependent on the phosphorylation status of $p R b 1^{18}$. The potential inhibitors of CDKs (p15INK4B, p16INK4A, p18INK4C and p19ARF), inhibit CDK4 and, in particular, CDK6, whereas p21WAF1/p27KIP1/p57KIP2 inhibitors halt the broad spectrum of various cyclins (including cyclin E) and cyclin-dependent kinases (CDK and CDK4/6) ${ }^{19}$. In addition, ubiquitin, by which CDK inhibitor p 27 is regulated $^{20}$, was also down-regulated in the ENU+EE treated rasH2 mice, whereas it was up-regulated in the ENU+EE treated ICR mice. Based on the results in the present study, we concluded that there is a tendency to slow down the cell cycle at the G1 to $\mathrm{S}$ transition phase in the ENU+EE treated rasH2 mice, whereas there is a strong tendency to accelerate the cell cycle in the ENU+EE treated ICR mice. Moreover, regarding the genes related to growth factors, in the ENU+EE treated rasH2 mice, several genes involved in growth factors such as EGF receptor, TGF beta receptor, and IGF were down-regulated, while these genes acted as stimulants of cell proliferation and carcinogenesis in the ENU+EE treated ICR mice.

In our previous report in which rasH2 and ICR mice were initiated with ENU followed by 2.5 ppm EE for 24 weeks, the PCNA positive indices for endometrial 
hyperplasias, atypical hyperplasias and adenocarcinomas in ICR mice were higher than those for luminal and endometrial glandular epithelia in which no proliferative lesions were developed in ICR mice, while the values for adenocarcinomas and atypical hyperplasias in the ENU alone group were comparable to those of the intact luminal and endometrial glandular epithelium in rasH2 mice ${ }^{6}$. These data may suggest a possible functional disorder of the cell cycle only in the uterine epithelium of rasH2 mice. However, with regard to the enhanced carcinogenesis in various organs of rasH2 mice treated with genotoxic carcinogens, it has been reported that somatic mutations of codons 12,13 and 61 of the c-Ha-ras transgene were detected in spontaneous or chemically induced tumors in rasH2 mice ${ }^{21-27}$. On the other hand, the incidences of point mutations of the transgene appeared to vary in tumors of rasH2 mice, and some carcinogens induced extremely low incidences of transgene mutations ${ }^{21,28,29}$. Thus, it can be considered that some factors other than the transgene mutation might be related to the tumor enhancement in rasH2 mice. It has been reported that c-Ha-ras mRNA gene expression in papillomas/squamous cell carcinomas of the forestomach induced by $\mathrm{N}$-methyl- $\mathrm{N}$-nitrosourea and lung adenomas induced by urethane in rasH2 mice were quantitated by means of the real-time RT-PCR, and overexpression of c-Ha-ras gene in the forestomach and lung tumors was found ${ }^{30}$. No mutations of endogenous Ha-ras, $\mathrm{Ki}-$ ras and $\mathrm{p} 53$ genes in mice were detected in rasH 2 mice, indicating that mutations of endogenous genes do not make a contribution toward carcinogenesis. Thus, it was proposed that overexpression of the transgene is the first important factor of the carcinogenesis in rasH2 mice rather than the transgene mutation. This explanation of the enhanced carcinogenesis in rasH2 mice is contradictory to the results of the present study and our previous study ${ }^{6}$.

In conclusion, we consider that the accelerated estrogen decomposition and the down-regulation of genes such as ER alpha, TGF beta, serine/threonine kinase and smad3, ubiquitin, EGF receptor, TGF beta receptor and IGF observed in the present study are strongly involved in the inhibition of uterine carcinogenesis in ENU-initiated rasH2 mice treated with EE. Since this is only a preliminary study, further analyses are needed to quantify the expression of these genes and identify additional genes involved in the inhibition of uterine tumorigenesis in ENU+EE treated rasH2 mice.

Acknowledgments: This work was supported in part by Grants-in-Aid for Cancer Research from the Ministry of Health, Labor and Welfare of Japan.

\section{References}

1. Mitsumori K, Furukawa F, Sato M, Yoshimura H, Imazawa T, Nishikawa A, and Takahashi M. Promoting effects of ethinylestradiol on development of renal proliferative lesions induced by $N$-nitrosobis(2-oxopropyl)amine in female Syrian golden hamsters. J Cancer Res Clin Oncol 1994; 120: 131-136.

2. Mitsumori K, Shimo T, Onodera H, Takagi H, Yasuhara K, Tamura T, Aoki Y, Nagata O, and Hirose M. Modifying effects of ethinylestradiol but not methoxychlor on $N$-ethyl$N$-nitrosourea-induced uterine carcinogenesis in heterozygous p53-deficient CBA mice. Toxicol Sci 2000; 58: $43-49$.

3. Vickers AE and Lucier GW. Estrogen receptor, epidermal growth factor receptor, and cellular ploidy in elutriated subpopulations of hepatocytes during liver tumor promotion by 17 alpha-ethinylestradiol in rats. Carcinogenesis 1991; 12: 391-399.

4. Watanabe T, Kashida Y, Yasuhara K, Koujitani T, Hirose $\mathrm{M}$, and Mitsumori K. Rapid induction of uterine endometrial proliferative lesions in transgenic mice carrying a human prototype c-Ha-ras gene (rasH2 mice) given a single intraperitoneal injection of $N$-ethyl- $N$-nitrosourea. Cancer Lett 2002; 188: 39-46.

5. Watanabe T, Kashida Y, Ueda M, Onodera H, Hirose M, and Mitsumori K. Promoting effects of ethinylestradiol but not atrazine on $N$-ethyl- $N$-nitrosourea-induced uterine carcinogenesis in ICR mice. J Toxicol Pathol 2003; 16: 139145.

6. Watanabe T, Kashida Y, Ueda M, Onodera H, Takizawa T, Hirose $\mathrm{M}$, and Mitsumori K. Inhibition by ethinylestradiol of $N$-ethyl- $N$-nitrosourea-initiated uterine carcinogenesis in transgenic mice carrying a human prototype c-Ha-ras gene (rasH2 mice). Toxicol Pathol 2003; 31: 496-505.

7. Lockhart DJ, Dong H, Byrne MC, Follettie MT, Gallo MV, Chee MS, Mittmann M, Wang C, Kobayashi M, Horton H, and Brown EL. Expression monitoring by hybridization to high-density oligonucleotide arrays. Nat Biotechnol 1996; 14: $1675-1680$.

8. Akhurst RJ and Balmain A. Genetic events and the role of TGF beta in epithelial tumour progression. J Pathol 1999; 187: 82-90.

9. Roberts $\mathrm{AB}$ and Derynck R. Meeting report: signaling schemes for TGF-beta. Sci STKE 2001; 113: PE43.

10. Ball $\mathrm{P}$ and Knuppen R. Catecholoestrogens (2-and 4hydroxyoestrogens): chemistry, biogenesis, metabolism, occurrence and physiological significance. Acta Endocrinol Suppl (Copenh) 1980; 232: 1-127.

11. Zhu BT and Conney AH. Functional role of estrogen metabolism in target cells: review and perspectives. Carcinogenesis 1998; 19: 1-27.

12. Cai XM, Conney AH, and Zhu BT. 17beta-estradiol metabolism by selectively-expressed human cytochromes P450. Proc Am Assoc Cancer Res 1998; 39: 2626.

13. Michalides R. Prognosis for G1 cell-cycle regulators: useful for predicting course of disease and for assessment of therapy in cancer. J Pathol 1999; 188: 341-343.

14. Milde-Langosch K and Riethdorf S. Role of cell-cycle regulatory proteins in gynecological cencer. J Cell Physiol 2003; 196: 224-244.

15. Bartek J, Bartkova J, and Lukas J. The retinoblastoma protein pathway in cell cycle control and cancer. Exp Cell Res 1997; 237: 1-6.

16. Collins K, Jacks T, and Pavletich NP. The cell cycle and cancer. Proc Natl Acad Sci USA 1997; 94: 2776-2778.

17. Semczuk A and Jakowicki JA. Alterations of pRb1-cyclin D 1-cdk4/6-p16(INK4A) pathway in endometrial 
carcinogenesis. Cancer Lett 2004; 203: 1-12.

18. Muller H, Lukas J, Schneider A, Warthoe P, Bartek J, Eilers $\mathrm{M}$, and Strauss M. Cyclin D1 expression is regulated by the retinoblastoma protein. Proc Natl Acad Sci USA 1994; 91: 2945-2949.

19. Sherr CJ and Robert JM. Inhibitors of mammalian G1 cyclin-dependent kinases. Genes Dev 1995; 9: 1149-1163.

20. Montagnoli A, Fiore F, Eytan E, Carrano AC, Draetta GF, Hershko A, and Pagano M. Ubiquitination of p27 is regulated by Cdk-dependent phosphorylation and trimeric complex formation. Genes Dev 1999; 13: 1181-1189.

21. Mitsumori K, Wakana S, Yamamoto S, Kodama Y, Yasuhara K, Nomura T, Hayashi Y, and Maronpot RR. Susceptibility of transgenic mice carrying human prototype c-Ha-ras gene in a short-term carcinogenicity study of vinyl carbamate and ras gene analyses on the induced tumors. Mol Carcinog 1997; 20: 298-307.

22. Sekiya T, Prassolov VS, Fushimi M, and Nishimura S. Transforming activity of the c-Ha-ras oncogene having two point mutations in codons 12 and 61. Jpn J Cancer Res 1985; 76: $851-855$.

23. Ando K, Saitoh A, Hino O, takahashi R, Kimura M, and Katsuki M. Chemically induced forestomach papillomas in transgenic mice carry mutant human c-Ha-ras transgenes. Cancer Res 1992; 52: 978-982.

24. Hayashi S, Mori I, Nonoyama T, and Mitsumori K. Point mutations of the c-Ha-ras genes in spontaneous liver tumors of transgenic mice carrying the human c-Ha-ras gene. Toxicol Pathol 1998; 26: 556-561.

25. Doi ST, Kimura M, and Katsuki M. Site-specific mutation of the human c-Ha-ras transgene induced by dimethylbezanthracene causes tissue-specific tumors in mice. Jpn J Cancer Res 1994; 85: 801-807.

26. Chin L, Tam A, Pomerantz J, Wong M, Holash J, Bardeesy N, Shen Q, O'Hagan R, Pantginis J, Zhou H, horner JW 2nd, Cordon-Cardo C, Yancopoulos GD, and DePinho RA. Essential role for oncogenic ras in tumor maintenance. Nature 1999; 400: 468-472.

27. Ogawa K, Imaida K, Masui $M$, Kawabe M, Hasegawa R, Kato K, Ito N, and Shirai T. Chemically induced lung and forestomach neoplasias in transgenic mice carry mutant forms of the human c-Ha-ras transgene. Carcinogenesis 1996; 17: 341-345.

28. Tsunematsu S, Saito H, Kagawa T, Morizane T, Hata J, Nakamura T, Ishii H, Tsuchiya M, Nomura T, and Katsuki $M$. Hepatic tumors induced by carbon tetrachloride in transgenic mice carrying a human c-Ha-ras proto-oncogene without mutations. Int J Cancer 1994; 59: 554-559.

29. Inoue R, Ushijima $T$, Katami M, Kushida H, Wakabayashi K, Sato H, Asamoto M, Katsuki M, Sugimura T, and Nagao M. Rare activation of the human c-Ha-ras transgene of mice in hemangioendothelial sarcomas and liver tumors induced by Glu-P-1. Jpn J Cancer Res 1996; 87: 583-588.

30. Maruyama C, Tomisawa M, Wakana S, Yamazaki H, Kijima H, Suemizu H, Ohnishi Y, Urano K, Hioki K, Usui T, Nakamura M, Tsuchida T, Mitsumori K, Nomura T, Tamaoki N, and Ueyama Y. Overexpression of human H-ras transgene is responsible for tumors induced by chemical carcinogens in mice. Oncol Rep 2001; 8: 233-237. 